\title{
A luciferase transgenic mouse model: visualization of prostate development and its androgen responsiveness in live animals
}

\author{
C-L Hsieh, Z Xie, Z-Y Liu', J E Green ${ }^{1}$, W D Martin², M W Datta², F Yeung ${ }^{3}$, D Pan ${ }^{3}$ and \\ L W K Chung \\ Molecular Urology and Therapeutics Program, Department of Urology, Emory University School of Medicine, 1365B Clifton Road, NE Suite B4100, Atlanta, Georgia 30322, \\ USA \\ ${ }^{1}$ Transgenic Oncogenesis Group, Laboratory of Cell Regulation and Carcinogenesis, National Cancer Institute, Bethesda, Maryland, USA \\ ${ }^{2}$ Department of Pathology, Emory University School of Medicine, Atlanta, Georgia, USA \\ ${ }^{3}$ University of Virginia School of Medicine, Charlottesville, Virginia, USA
}

(Requests for offprints should be addressed to C-L Hsieh; Email: chsieh2@emory.edu)

\begin{abstract}
Numerous mouse models of prostate carcinogenesis have been developed, but hitherto there has been no model in which the prostate gland could be imaged in live animals. The transgenic model generated here targeted mouse prostate gland using a firefly luciferase enzyme under the control of a small but highly active and specific supra prostate-specific antigen (sPSA) promoter. We evaluated postnatal prostate development, involution and androgeninduced restoration of prostate growth in adult transgenic mice using bioluminescence imaging. Results of our study showed that: (i) the prostate gland of male offspring did not yield a significant bioluminescence signal until after sexual maturity. Luciferase was detected in the luminal epithelial cells of the ventral and dorsolateral lobes of the prostate gland and caput epididymis, with little or no activity in 18 other organs evaluated. (ii) While a constant high level of bioluminescence was detected in the mouse prostate from 5 to 35 weeks of age, a slight drop in bioluminescence was detected at 36 to 54 weeks. (iii) Upon castration, the luciferase activity signal associated with mouse prostate detected by a cooled charge-coupled device camera was dramatically reduced. This signal could be rapidly restored to pre-castration levels after androgen administration. Androgen-induced luciferase activity subsided to nearly basal levels 5 days following the last injection. These data demonstrate that a bioluminescent mouse model with luciferase activity restricted to the prostate gland under the control of a (SPSA) promoter can be used on a real-time basis in live animals to investigate the development and responsiveness of the prostate gland to exogenously administered androgen. This model can be extended to detect the responsiveness of the prostate gland to therapy and used as a founder strain to visualize tumors in hosts with different genetic backgrounds.
\end{abstract}

Journal of Molecular Endocrinology (2005) 35, 293-304

\section{Introduction}

Classic investigations of prostate development and prostate response to androgen have commonly used animals killed after appropriate treatment (Chung \& Auble 1988, Leav et al. 2001, Putz et al. 2001). The results of these types of studies are susceptible to individual variations among mouse strains regardless of genetic background, potential effects of experimental manipulation on tissues harvested from animals, and a lack of real-time information during the study. In the quest for improved experimental models, we developed a luciferase-tagged transgenic mouse strain with a bioluminescent marker expressed specifically in the prostate gland of the male mouse. Human prostatespecific antigen (PSA) gene promoter is a logical choice to target prostate genes in transgenic mice, since PSA protein is selectively expressed by the human prostate. The specificity of the luciferase enzyme expression in the mouse prostate was achieved by using a potent supra PSA (sPSA) promoter, constructed by linking a $440 \mathrm{bp}$ androgen responsive element enhancer core (AREc) that encompasses the AREIII region and a $150 \mathrm{bp}$ upstream fragment of the TATA box, $\mathrm{pN} / \mathrm{H}$ of the full-length of PSA promoter (Yeung et al. 2000). sPSA promoter retained tissue specificity but exhibited 2- to 3-fold higher promoter activity than full-length PSA promoter when tested in human prostate cancer cell lines (Yeung et al. 2000). Consistent with our previous demonstration (Yeung et al. 2000), sPSA promoter, like full-length PSA promoter, can drive the expression of a transgene specifically in the mouse prostate gland. The goals of this study were to test whether sPSA promoter retained prostate specificity in a transgenic animal model by 
following the expression of luciferase as the transgene, and whether this sPSA transgenic mouse model could be used to evaluate prostate development and the responsiveness of the prostate gland to androgen deprivation and exogenously administered androgen.

Luciferase reporter gene expression in live animals can be measured with a cooled charge-coupled device (GCCD) camera minutes after the administration of luciferin. The low background of luminescence from normal tissue, the rapid turnover of luciferase enzyme, and the non-immunogenic characteristics of luciferin make this method ideally suited for temporal in vivo imaging of gene expression. Recently, this strategy was used successfully for the non-invasive detection of transplanted tumors by ex vivo (Contag et al. 1998, Edinger et al. 1999, Sweeney et al. 1999, Rehemtulla et al. 2000, Zhang et al. 2002) and in vivo gene delivery (Honigman et al. 2001, Wu JC et al. 2001, 2002) in mice. Consecutive bioluminescent images acquired from the same animals provide temporal and spatial information throughout an entire experiment, which has benefited the study of tumor progression (Edinger et al. 1999, Adams et al. 2002, Vooijs et al. 2002), antineoplastic therapies (Rehemtulla et al. 2000, 2002), and promoter activation (Wu N et al. 2002, Zhang et al. 2003). Here we report the generation of a transgenic mouse model that enables non-invasive bioluminescent imaging of prostate gland development and assessment of androgen responsiveness of the prostate gland to castration and androgen replacement by overexpressing luciferase gene in the prostate gland under the control of sPSA promoter. This model also confirmed the specificity of sPSA promoter for transgene delivery to the prostate gland.

\section{Materials and methods}

\section{Generation and identification of transgenic mice}

To develop transgenic sPSA-Luc mice, the plasmid sPSA-Luc was linearized with $\mathrm{KpnI}$ and BamHI. The $2.6 \mathrm{~kb}$ fragment of sPSA-Luc, which contained the sPSA promoter, the luciferase reporter gene and the SV40 poly (A) signal, was purified by gel electrophoresis and prepared for injection according to standard methods (Sambrook 1989). The appropriate fragments were microinjected into the male pronuclei of fertilized eggs of FVB/N mice (Taconic Co., NY, USA) (Hogan 1994). Transgenic founders were identified by Southern blot analysis of tail-tip DNA. Briefly, $10 \mu \mathrm{g}$ genomic tail DNA isolated by standard procedures were digested with $\mathrm{Xho}$ and $\mathrm{XbaI}$ and then fractionated by $1 \%$ agarose gel electrophoresis, and transferred to a Hybond NX (Amersham Pharmacia Biotech) membrane. The blots were probed with a ${ }^{32}$ P-labeled sPSA-Luc fragment obtained by digesting
sPSA-Luc/pGL-3 with EcoRV and SphI. After founders were established, genotyping of offspring was characterized by PCR of purified tail DNA $(\sim 50 \mathrm{ng})$. The screening primers used for PCR were sPSA forward-as: 5'CAGAACGATGGAGAATTG3' and luciferase reverse-as: 5'CAAGTGGAACTCGGATAA3' (indicated in Fig. 1a). Cycling parameters were an initial denaturation at $95^{\circ} \mathrm{C}$ for $5 \mathrm{~min}$ followed by 35 cycles with each cycle at $94{ }^{\circ} \mathrm{C}$ for $1 \mathrm{~min}, 53{ }^{\circ} \mathrm{C}$ for $1 \mathrm{~min}$ and $72{ }^{\circ} \mathrm{C}$ for $1 \mathrm{~min}$ and the last extension at $72{ }^{\circ} \mathrm{C}$ for $7 \mathrm{~min}$. The $550 \mathrm{bp}$ PCR products $(8 \mu \mathrm{l})$ were separated by $1 \%$ agarose gel electrophoresis. For semi-quantitative determination of the copy number of transgenes, different amounts of purified sPSA-Luc fragment DNA obtained from sPSA-Luc plasmid (from $0-10^{7}$ relative copies) and mouse tail DNA (from $10^{2}-10^{4}$ copies) were performed by PCR as described above in parallel. The intensity of PGR products from mouse DNA was quantified by scanning densitometry (Quantity one- $4 \cdot 1 \cdot 1$ Gel Doc gel documentation; Bio-Rad) and compared with those from sPSA-Luc plasmid DNA. The copy number of transgenes per diploid genome of each transgenic mouse was determined by comparing the relative copy number of transgenes with that of genomic DNA.

\section{Luciferase enzymatic assay}

Organs were harvested from transgenic mice and assayed for luciferase activity. Briefly, organ tissue was homogenized in an appropriate amount of cell lysis buffer and then centrifuged for 3 min. Both luciferase activity and protein concentration in supernatants were determined using the Luciferase Reporter Assay System (Promega) and BCA Protein Assay Reagent (Pierce) respectively. For luciferase activity detection, $20 \mu \mathrm{l}$ supernatant were mixed with $100 \mu \mathrm{l}$ luciferase substrate as described by the reporter assay system, and luciferase activity was quantified in a Monolight 3010 luminometer (BD PharMingen). The results are reported as relative light units per microgram total protein.

\section{In vivo bioluminescence imaging}

In vivo bioluminescence imaging was conducted on a cryogenically cooled IVIS system (Xenogen Corp., CA, USA) using LivingImaging acquisition and analysis software (Xenogen). Briefly, mice were anesthetized with ketamine/xylazine/acepromazine and subsequently received an i.p. injection of an aqueous solution of the substrate D-luciferin $(125 \mathrm{mg} / \mathrm{kg})$. The animals were then placed in a light-tight chamber and imaged with a CGCD camera. Images were acquired $25 \mathrm{~min}$ after luciferin administration. An integration time of $1 \mathrm{~min}$ with a binning of 100 pixels was used for luminescence image acquisition. Signal intensity was quantified as the 


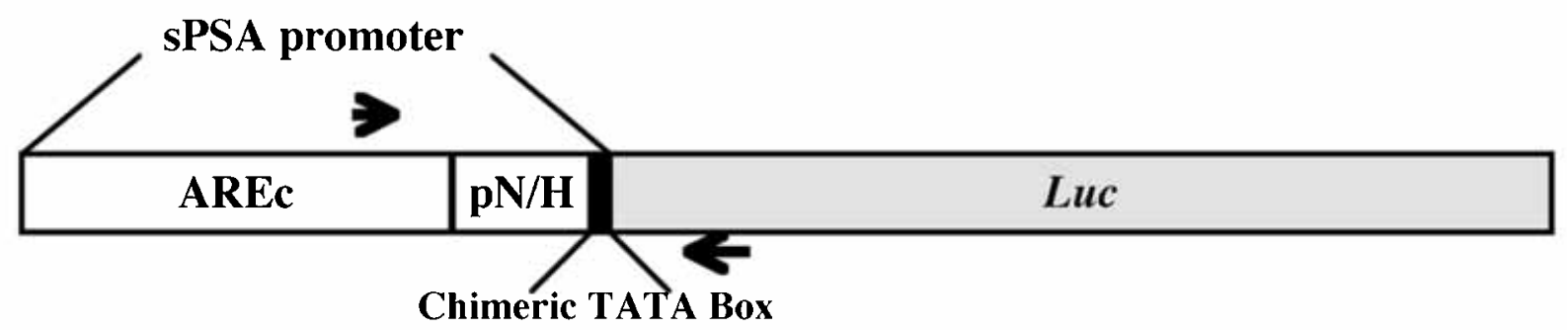

b

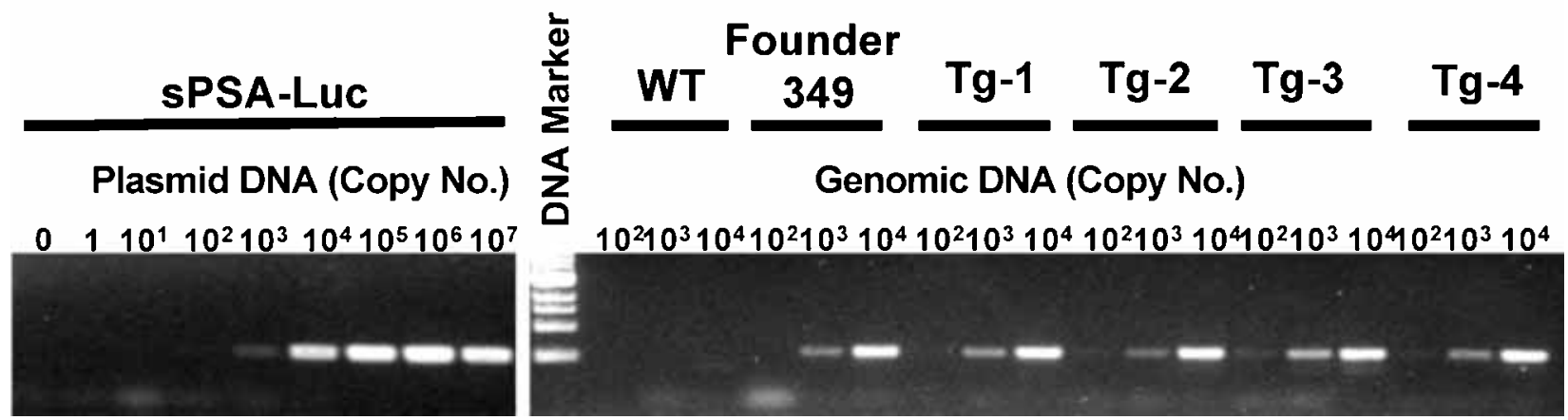

Figure 1 Genotyping the sPSA-Luc transgenic FVB/N mouse strain. (a) Structure of the sPSA-Luc transgene. Arrow indicates location of screening primer for genotyping by PCR. (b) Semi-quantitative determination of the copy number of transgenes by PCR analysis. Purified SPSA-Luc fragment DNA obtained from SPSA-Luc plasmid (from 0-107 relative copies) and mouse tail DNA $\left(10^{2}-10^{4}\right.$ copies) from wild type (WT), founder (Founder 349), and transgenic (Tg-1 to Tg-4) mice was performed by PCR as described in the Materials and methods. The intensity of serially diluted mouse genomic DNA was compared with different amounts of SPSA-Luc plasmid DNA (from $0-10^{7}$ relative copies as indicated).

sum of all detected photon counts within the region of interest after subtraction of background luminescence.

\section{Luciferase immunohistochemical (IHC) detection}

Organs harvested from wild-type (WT) or transgenic mice were fixed in $10 \%$ buffered formalin, embedded in paraffin, and sectioned onto glass slides. Paraffinembedded sections were hydrated through xylene and graded alcohol and equilibrated in PBS. Antigen retrieval was performed by heating the slides in $10 \mathrm{mM}$ sodium citrate $(\mathrm{pH} 6.0)$ at $110{ }^{\circ} \mathrm{C}$ for $4 \mathrm{~min}$ followed by staining for luciferase with monoclonal mouse antiluciferase antibody (Clone LUC-1; Sigma) in a 1:50 dilution. Staining was performed using EnVision Plus Systems (Dako) according to the manufacturer's protocol.

\section{Experimental animals}

In accordance with the NIH Guidelines for Care and Use of Laboratory Animals, all experiments were conducted using the highest standard of humane care.

\section{Results}

\section{Generation of transgenic mice}

Transgenic mice were generated using the sPSA-Luc construct, which contains $590 \mathrm{bp}$ of sPSA promoter sequences linked to a chimeric TATA box and the $1.7 \mathrm{~kb}$ of firefly luciferase coding region (Fig. la). Three sPSA-Luc founder animals were identified by Southern blot analyses (data not shown). Founder mice were backcrossed to FVB/N mice to generate progeny. The protocols were approved by the Emory University Animal Care and Use Committee, Protocol No. 163-2002. One male founder died very early at the beginning of the mating period and another male founder was infertile. The remaining female founder line (designated as founder 349) showed transmission of the transgene to their offspring with a consistency of approximately two to five copies of transgenes per diploid genome of the transgenic mice, as determined by semi-quantitative PCR analysis comparing transgenic bands with the copy number control of the plasmid (Fig. 1b). Homozygous transgenic mice were used in the 
following quantitative studies to minimize genotypic variability.

\section{Transgene distribution in PSA-Luc mice}

To determine the expression pattern of the transgene in vivo, bioluminescent imaging was performed in living animals including non-transgenic littermates and the sPSA-Luc line at 10-16 weeks of age. To ensure that the distribution of luciferin in the various organs is not rate-limiting for luciferase activity, the optimal time for detection of light emission from the transgenic animals was determined by whole-body imaging from three homozygous transgenic males at different time intervals following systemic injection of the luciferin. All tested mice displayed similar kinetic profiles while the peak of light emission was reached between 20 and $30 \mathrm{~min}$ after injection of luciferin (Fig. 2a). Hence, in all of the experiments described below, bioluminescent imaging was monitored $25 \mathrm{~min}$ after administration of luciferin.

All the sPSA-Luc male mice we tested displayed a hot spot of light emission exclusively in the lower abdomen, whereas no background signal was detected in non-transgenic mice (Fig. 2b). A few sPSA-Luc mice occasionally showed a very weak signal located on the feet or tail in both sexes (data not shown). The whole-body light emissions quantified from sPSA-Luc males were 158-fold higher than those from the sPSA-Luc females (Fig. 2b). To localize the origin of the luminescence, we isolated organs from the mice and re-imaged them. The signals in the lower abdomen were found to originate from the prostate and caput epididymis, and the rare signal detected in the tail was from the bone (Fig. 2c). The luciferase signal in the prostate was 10- and 166-fold relative to the epididymis and the tailbone respectively. No light was emitted from the mouse after removal of these organs (data not shown).

To confirm in vivo bioluminescent imaging, luciferase activity was also investigated in vitro on a panel of tissues isolated from the imaged mice. Figure $3 \mathrm{a}$ shows that the ventral lobe of the prostate gland displayed the highest luciferase activity. Dorsolateral prostate luciferase activity was approximately $10 \%$ of that found in the ventral prostate, and the anterior prostate had less. A moderate level of luciferase activity was detected in the caput epididymis but none in the cauda epididymis. Other organs, including seminal vesicle, testis and salivary gland, reported as non-prostate target tissues of other prostate-specific promoters (Maroulakou et al. 1994, Schaffner et al. 1995), showed no luciferase activity in our sPSA-Luc mouse line. However, both male and female mice expressed a relatively low level of luciferase activity in the bone marrow, without significant differences between sexes. IHC staining with luciferasespecific antibody verified tissue and cellular distribution of this enzyme. Figure 3b shows that, consistent with both in vivo bioluminescence imaging and in vitro luciferase assay, the strongest luciferase staining was seen in the ventral prostate with lower levels in the dorsolateral prostate. The staining was present in approximately $90 \%$ of the luminal epithelial cells examined. No basal cells or stromal cells displayed positive staining.

\section{Developmental regulation of sPSA promoter in transgenic mouse}

We investigated whether bioluminescence could be used to assess the dynamic response of sPSA promoter to testicular androgen and growth factors in the microenvironment during mouse prostate development. In vivo imaging of individual sPSA-Luc mice was analyzed weekly for over 1 year. Targeting of transgene expression in the prostate was not found in male mice aged 2 to 4 weeks but luciferase activity was markedly elevated at the onset of sexual maturity. Unexpectedly, luciferase activity was detected in leg and tail initially but light emission decreased with age and declined to background activity when male (nine out of ten) and female (six out of six) mice reached puberty (Fig. 4a). The leg and tail bioluminescence signals were confirmed to originate from bone marrow as assessed by luciferase assay and IHC staining (data not shown). Measurements of mice between 5 and 35 weeks old showed constant and high bioluminescence levels $\left(4 \cdot 25 \pm 2 \cdot 1 \times 10^{7}\right.$ photons/s), an indication of active androgen production and increased efficiency of androgen-induced gene transcription and translation (Zhang et al. 2003). From 36 to 50 weeks of age, a somewhat lower bioluminescence level $\left(1 \cdot 5 \pm 0 \cdot 8 \times 10^{7}\right.$ photons/s $)$ was observed. The signal dropped to the background level when the mice were 56 weeks old (Fig. 4b), reflecting decreased androgen production in aged males (Bronson \& Desjardins 1977, Xie et al. 2004).

\section{In vivo hormone response of the sPSA promoter}

To verify the androgen responsiveness of the sPSA promoter in transgenic mice, sexually mature males (aged 10-12 weeks) were castrated, followed 7 days later by dihydrotestosterone (DHT) or vehicle (sesame oil) administration. The bioluminescence signal correlated well with androgen withdrawal and an androgen supplement restored luciferase activity in the prostate (Fig. 5a). The intact sPSA-Luc male mice typically emitted $>10^{7}$ photons/s in the prostate gland (Fig. 2b). Castrations led to an approximately 100-fold decrease in the imaging signal by day 4 , and then dropped to the background level by day 7 (Fig. 5b). Injection of DHT at day 7 produced a significant induction of the imaging signal, rising rapidly to pre-castration (day 0) levels within 1 day (day 8) and then dropping slightly on day 9 . DHT injection on day 9 was found to restore luciferase 
a

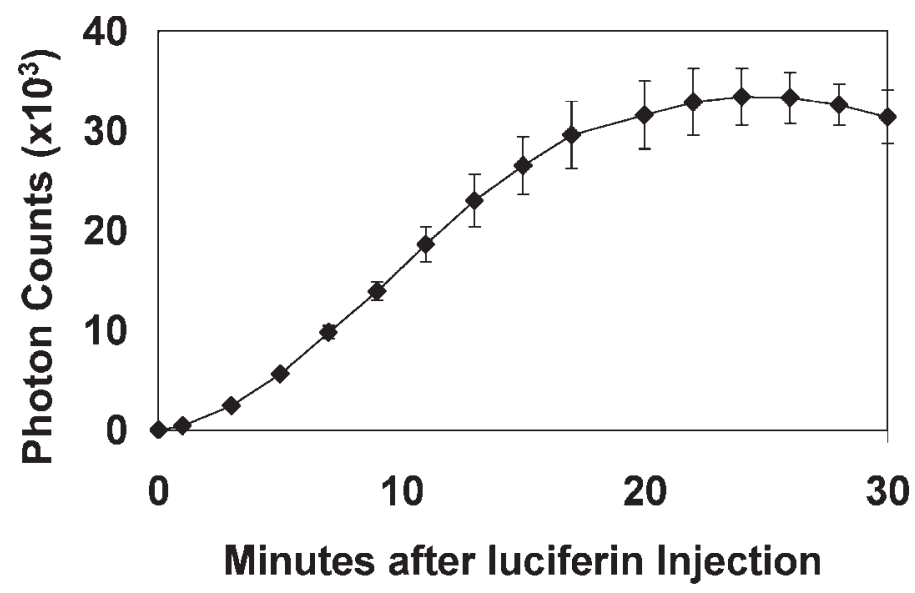

b

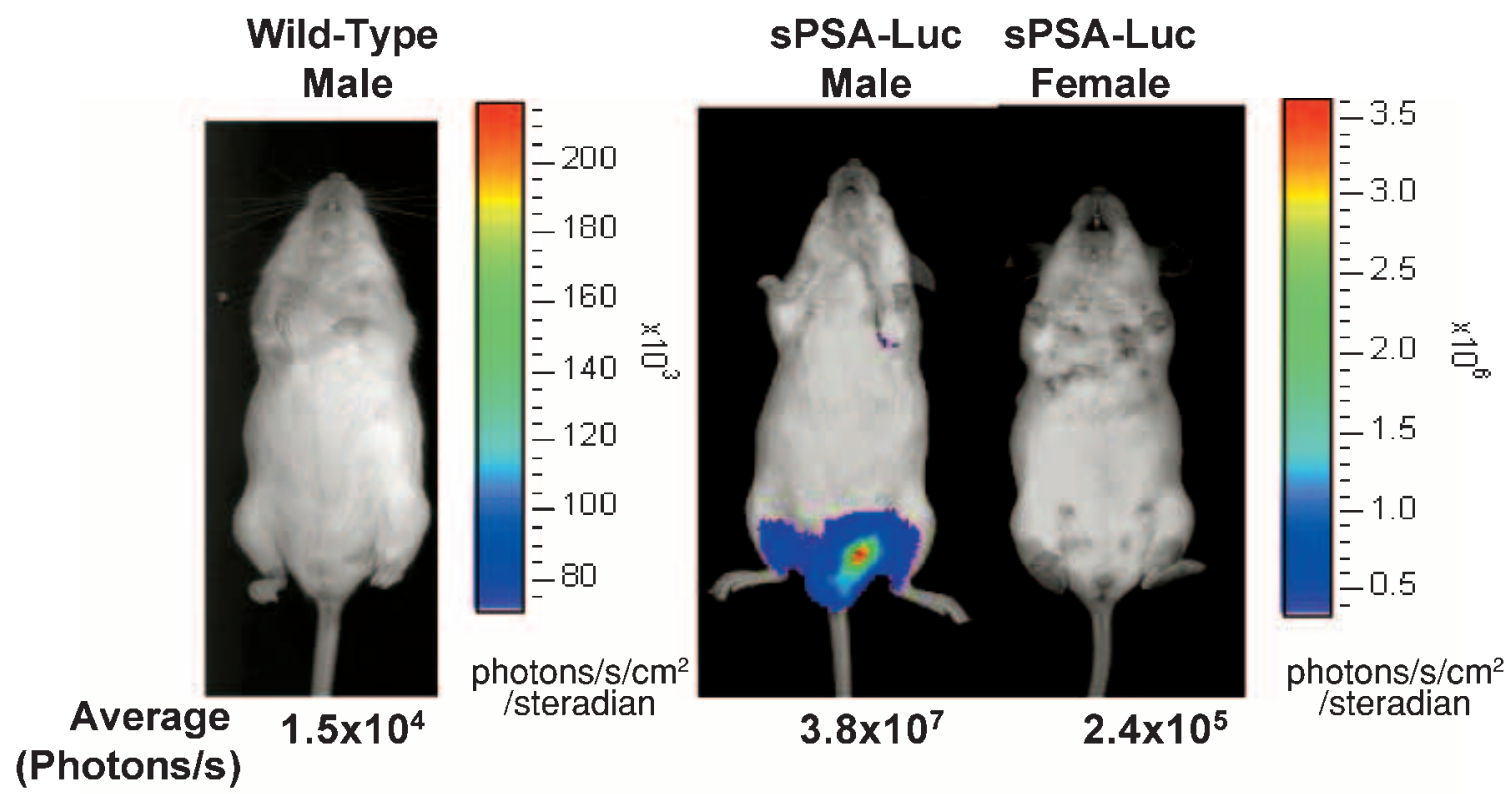

C

Prostate

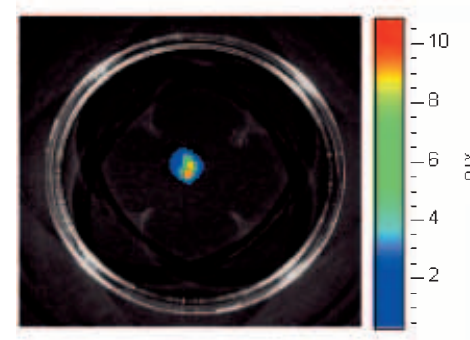

$2.33 \times 10^{7}$
Epididymis

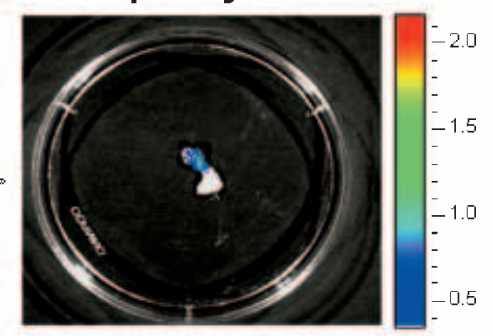

$2.5 \times 10^{6}$

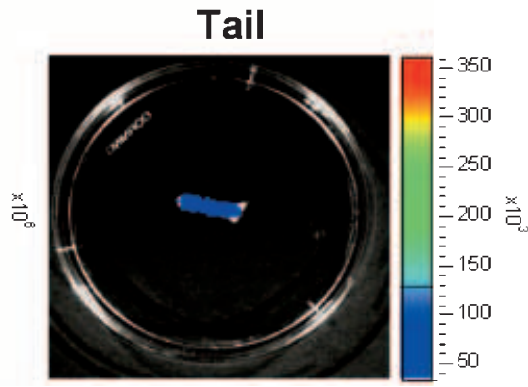

$1.4 \times 10^{5}$

Photons/second 

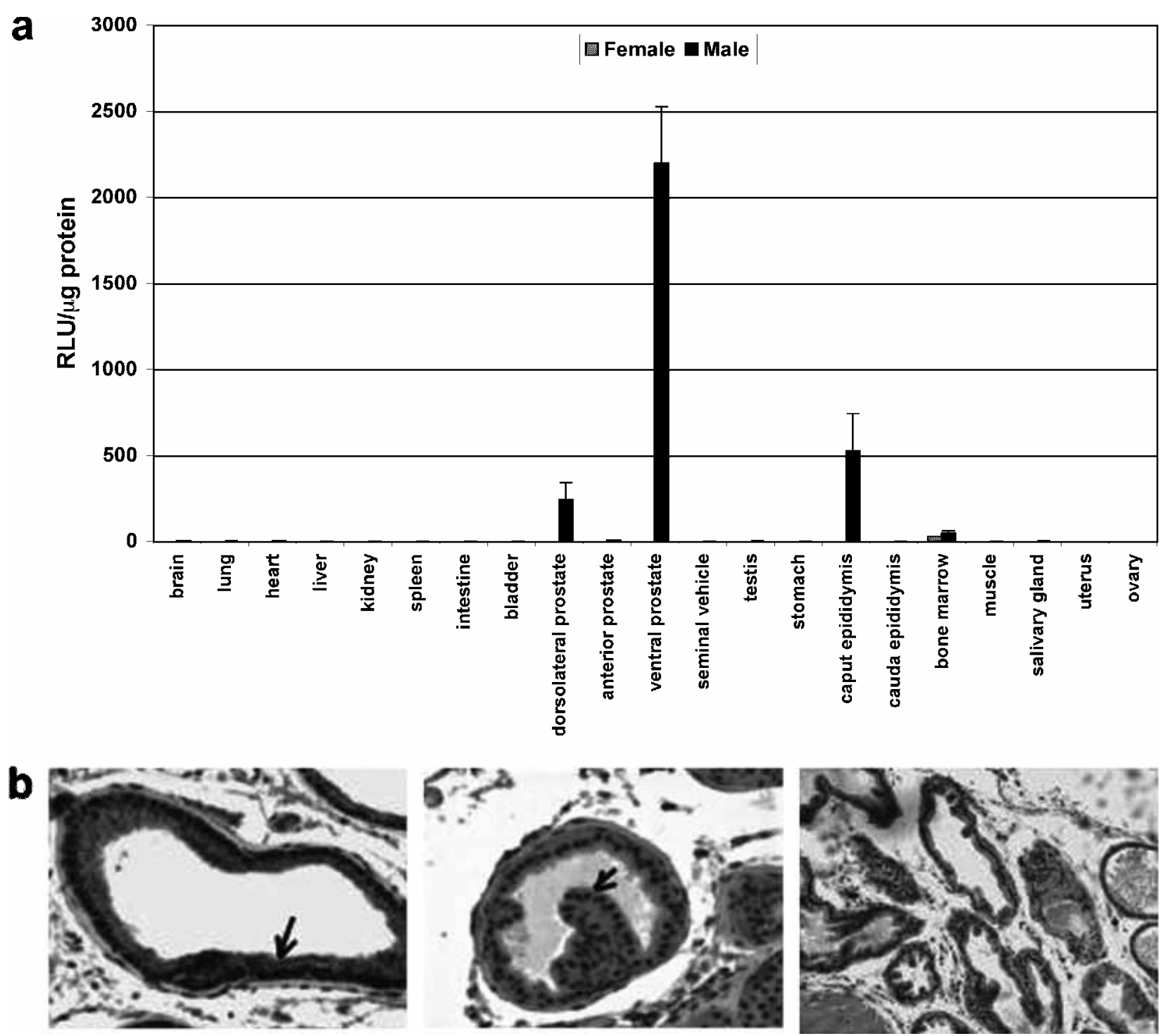

Figure 3 Tissue distribution of luciferase activity. (a) luciferase assay of tissue extracts from 10- to 16-week-old sPSA-Luc male and female mice using a luminometer. Data are presented as relative light units (RLU) per microgram of protein and shown as means of six mice \pm S.D. (b) IHC of luciferase expression in the ventral prostate (left panel) and dorsolateral prostate of sPSA-Luc mice (middle panel). Expression of luciferase protein in the luminal epithelial cells is indicated. Original photograph at $\times 400$ magnification. A section of the prostate from non-transgenic littermate (right panel) shows negative immunoreactivity ( $\times 100$ magnification).

activity detection on day 10 . A continuous drop in luciferase activity was observed and reached background level on day 14. In the same study, dexamethasone injected at the same dose had no effect on the induction of bioluminescence in castrated mice (data not shown), confirming the specificity of sPSA promoter reporter, which responded exclusively to androgen. This is consistent with the fact that the sPSA promoter sequence contains only ARE and is devoid of glucocorticoid response element (GRE).

Figure 2 Bioluminescence imaging of luciferase activity in sPSA-Luc transgenic mice. (a) Kinetics of light emission following injection of luciferin in transgenic males at 10-12 weeks of age. Total signal intensity (photon counts) over the whole body was determined and plotted with respected to time. Data presented are means of three mice \pm S.D. (b) Ventral view of images of representative SPSA-Luc transgenic male and female, and non-transgenic littermate male mice (wild-type). The color scale next to the images indicates the signal intensity at the surface of the animals in photon/s. (c) Isolated organs from a 12-week-old sPSA-Luc male mouse were imaged. The quantified photon signal is shown below each image. 
a

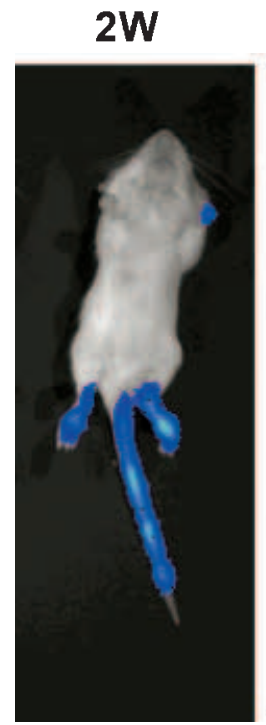

$3 W$

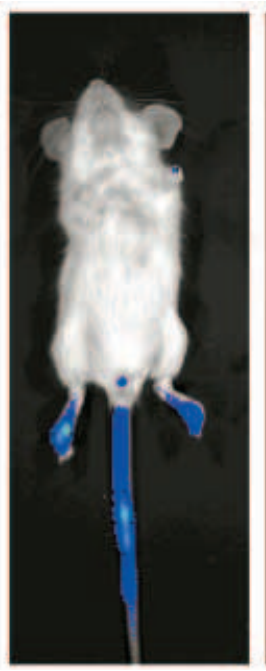

4W

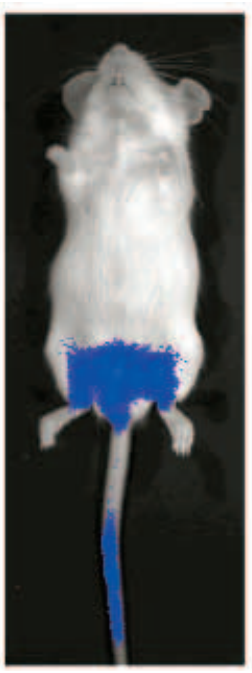

$5 W$

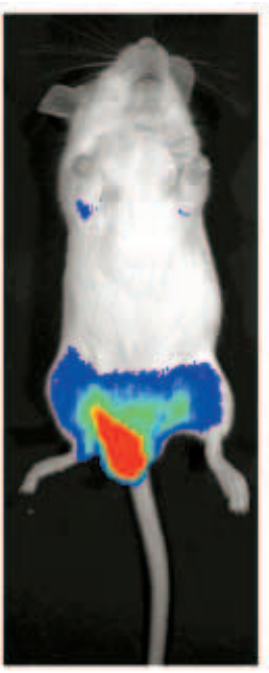

\section{$6 W$}

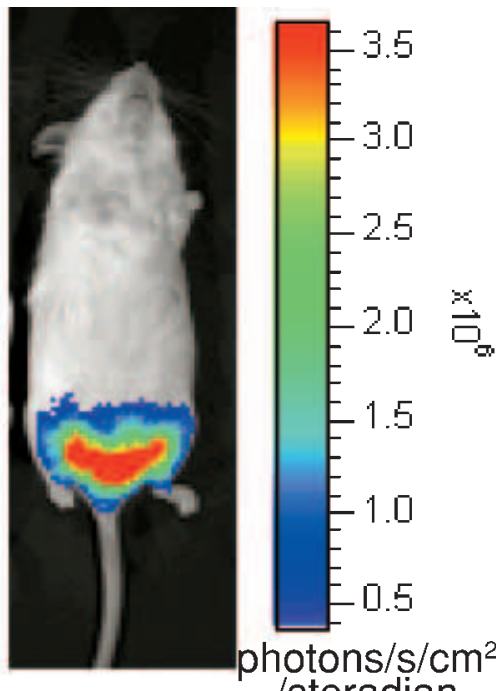

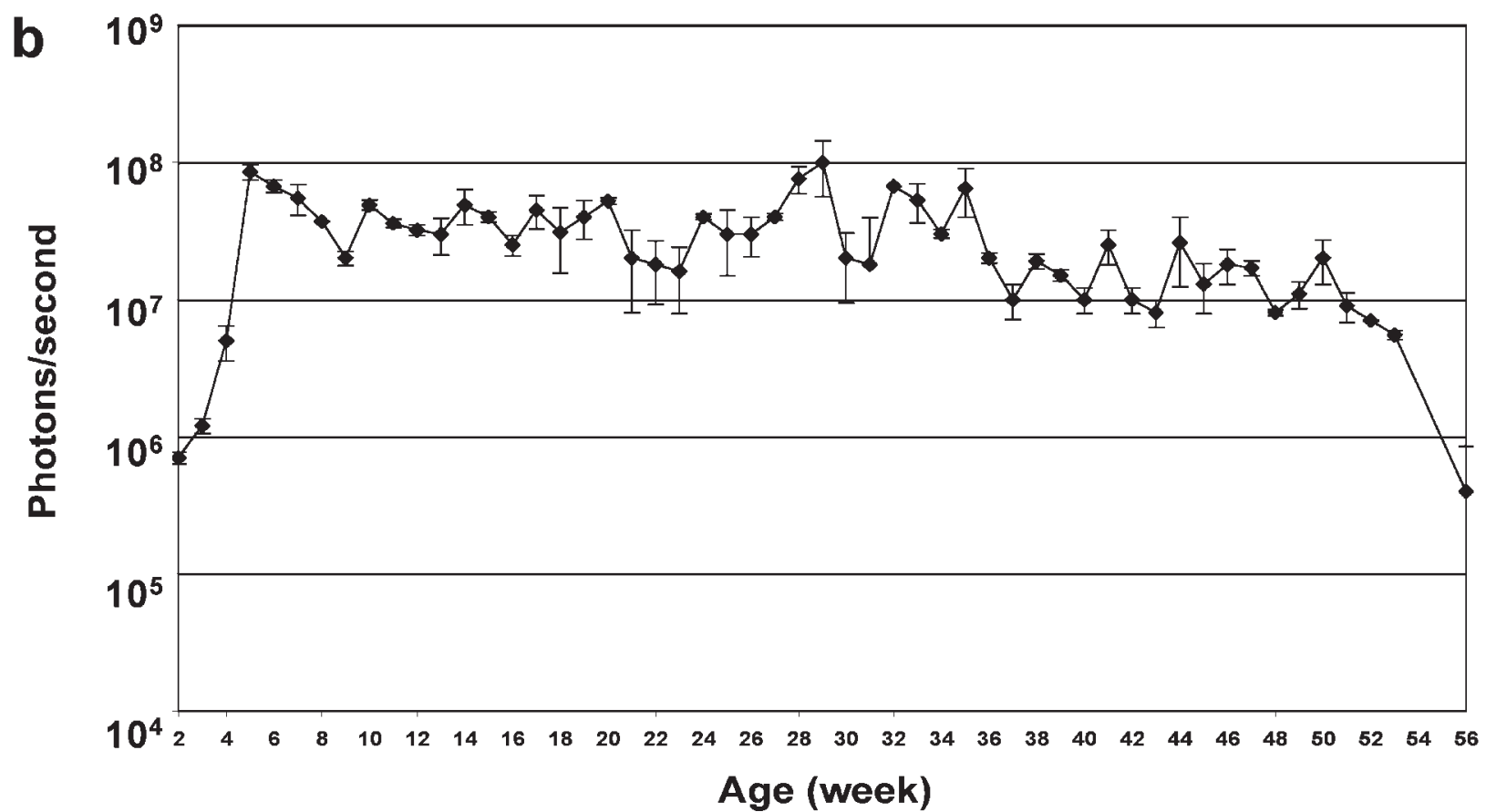

Figure 4 Developmental regulation of sPSA-Luc expression. (a) Bioluminescent images of a representative individual mouse. The animals were measured at 1 -week intervals from 2 to 6 weeks (W) old. Signals were adjusted to the same color scale for the entire time course. (b) quantification of photons emitted from the lower abdomen of sPSA-Luc male mice from 2 to 56 weeks old. Data are presented as average photon count/s of three mice S.D.

\section{Discussion}

A novel bioluminescent transgenic mouse model, sPSA-Luc, with firefly luciferase enzyme expression restricted to the prostate gland under the control of a potent s-PSA promoter, was generated and character- ized (Figs 2 and 3). This mouse model was used in a developmental study to assess endogenous androgen regulation of target genes in the prostate gland as a function of age (Fig. 4) and to confirm the activity of exogenously administered androgen, which regulates sPSA promoter-luciferase reporter activity in the 


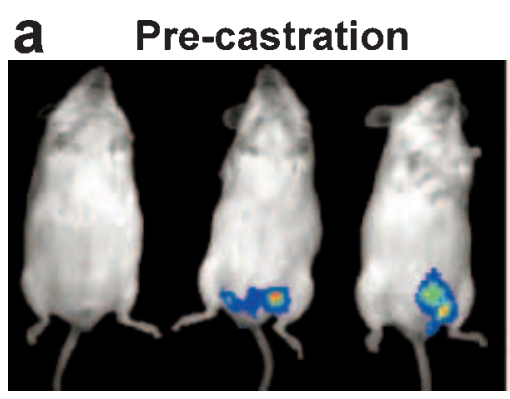

WT Tg-1 Tg-2

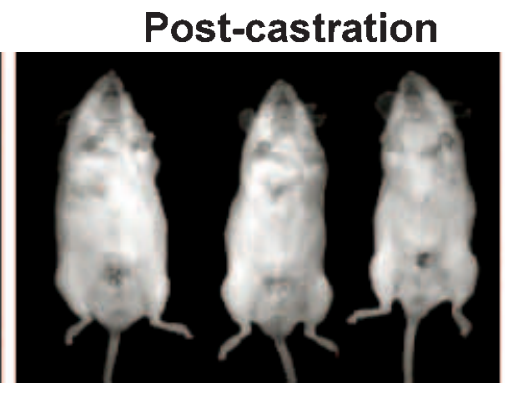

WT

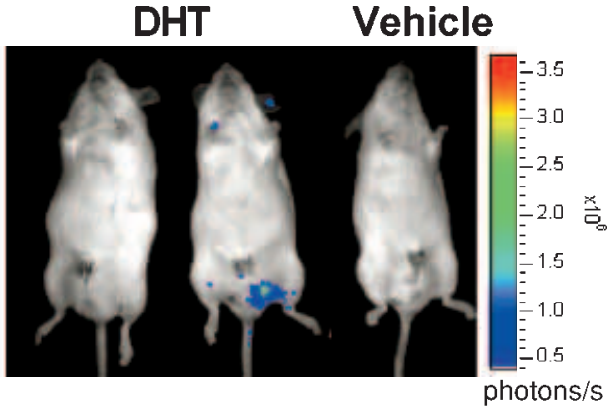

WT Tg-1

Tg-2 $/ \mathrm{cm}^{2}$ /steradian

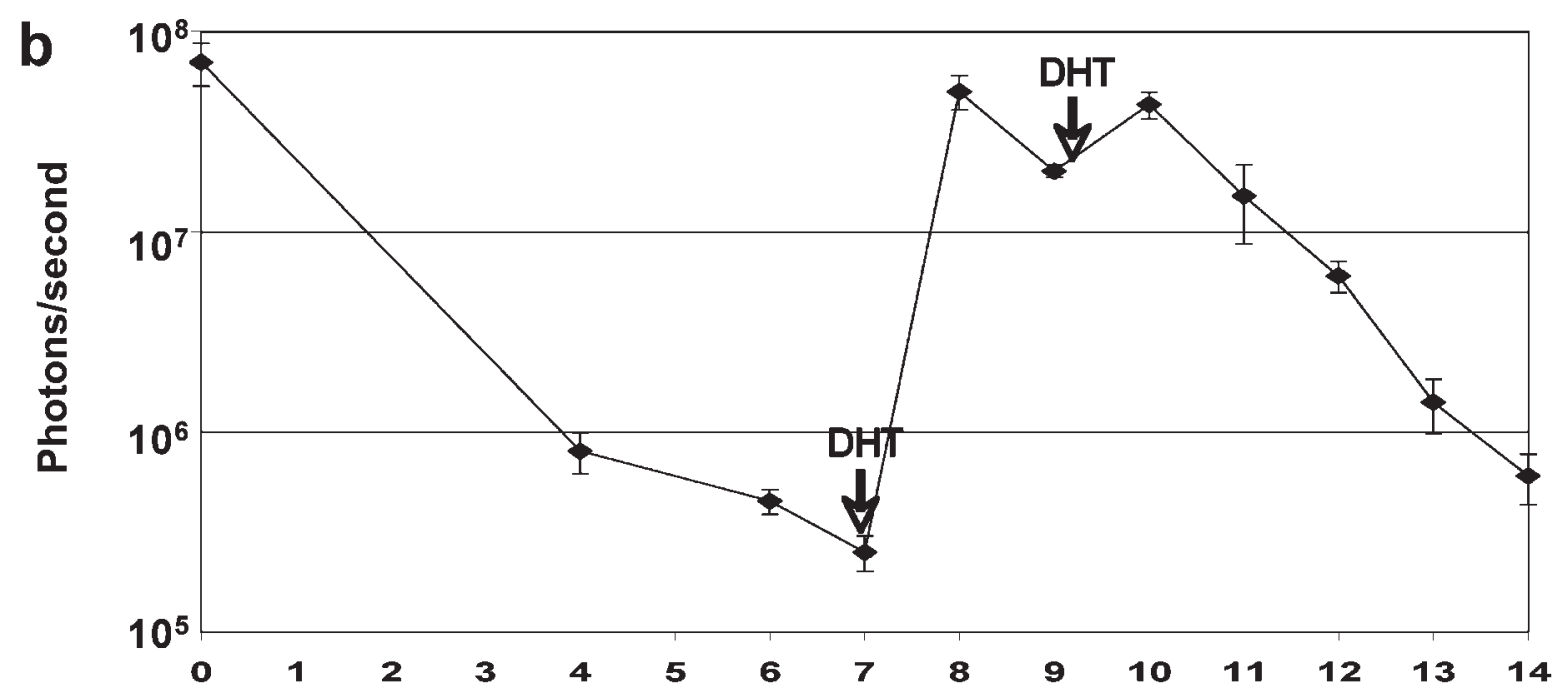

\section{Days after Castration}

Figure 5 Androgen regulation of sPSA-Luc expression. (a) Bioluminescent images of mice during the pre-castration, post-castration and DHT-treatment period. Mice were castrated at 10 weeks of age. After 7 days, some mice were supplemented with DHT ( $5 \mathrm{mg} / \mathrm{kg}$ body weight) or vehicle (sesame oil) i.p. at the indicated time point. Non-transgenic littermates (WT) served as control. (b) The signals from a representative transgenic mouse were quantified and plotted as photons/s during the treatment protocol.

prostate gland of live animals (Fig. 5). Using the same animals for repeated measurement at either different ages (from 2 to 56 weeks) or hormonal status (castration vs castration followed by androgen replacement), we demonstrated the specificity of the androgen regulation of sPSA promoter-luciferase reporter activity in the developing mouse prostate and in mice under the influence of exogenously administered androgen. Our results are in agreement with numerous previous studies (Allison et al. 1989, Greenberg et al. 1994, 1995, Perez-Stable et al. 1996, Garabedian et al. 1998, Kasper et al. 1998, Zhang et al. 2000, Gabril et al. 2002) defining androgen regulation of other target genes in the developing mouse prostate gland. Unlike previous studies, however, our results provided a continuous real-time measurement of the bioluminescence emitted from the luciferase reporter, which was under the control of a well-known androgen receptor (AR)regulated promoter, sPSA gene (Yeung et al. 2000). Based on luciferase imaging, we demonstrated the exquisite specificity of sPSA promoter in directing high level and luminal epithelial-specific expression of luciferase enzyme in the ventral lobe of the mouse prostate gland. A moderate to low level of luciferase activity was detected in mouse caput epididymis and bone marrow respectively. No luciferase reporter activity was detected in seminal vesicle, testis and salivary gland, which have frequently been reported to express other prostate-targeting promoters in transgenic mouse models (Maroulakou et al. 1994, Schaffner et al. 1995). Although PSA is not expressed in mouse prostate or mouse kallikrein genes identified to date by shared homology 
with human PSA (Clements 1989), the specificity of sPSA promoter in regulating downstream luciferase reporter expression largely in the mouse ventral prostate gland as demonstrated herein suggests a high degree of conservation of transcriptional factors in the prostate gland between mouse and human. This interpretation is consistent with our previous transgenic mouse study using a $6 \mathrm{~kb}$ fragment of the PSA promoter-LacZ construct (Cleutjens et al. 1997b), suggesting that the $590 \mathrm{bp}$ sPSA promoter contains most, if not all, information for prostate-specific and androgen-regulated activity. It is also in agreement with previous in vitro studies conducted by our laboratory (Yeung et al. 2000) and others (Schuur et al. 1996, Cleutjens et al. 1997a) showing that the $440 \mathrm{bp}$ AREc enhancer is sufficient to cooperate with a minimal promoter to confer prostatespecificity and androgen-responsiveness.

The mouse prostate gland is known to consist of several lobes, differing markedly from the zonal anatomical structures and pathology of the human prostate gland. Previous publications suggest a certain structural homology between mouse and human prostate gland based on embryological development and morphological criteria (Price 1963, Steers 2001, Vaarala et al. 2001, Marker et al. 2003, Shappell et al. 2003). To date, however, there is no existing supporting evidence for a direct relationship between mouse prostate lobes and specific zones in the human prostate (Shappell et al. 2004) based on anatomical considerations. The mechanisms by which human prostate-specific promoters specifically direct transgene expression to the mouse prostate are not understood. Even with PSA promoterdirected transgene expression, one $6 \mathrm{~kb}$ full-length and the other $590 \mathrm{bp}$, we observed similar prostate specificity but a marked difference in lobular distribution of transgene with the $6 \mathrm{~kb}$ PSA promoter-directed $\beta$-galactosidase expressed in the mouse dorsolateral lobe (Cleutjens et al. 1997b) and the 590 bp sPSA promoteractivated luciferase enzyme detected almost entirely in the ventral lobe of the prostate gland. Interestingly, the ventral lobe specificity of the transgene expression directed by sPSA promoter corresponded to the expression of PSA in a transgenic mouse strain established by the introduction of human PSA under the control of a homologous human PSA genomic DNA (Wei et al. 1997). A similar pattern of transgene expression was also reported in another prostate-specific transgenic mouse model using human kallikrein 2-based promoter (Xie et al. 2004). These data collectively suggest a strong homology between human prostate and the ventral lobe of mouse prostate with respect to PSA regulation. Whether or how the differential and lobular differences in PSA regulation relate to prostate cancer induction and subsequent progression in mouse models, and their relationship to human prostate, will be an interesting subject for future investigation.
Other than the prostate gland, the published literature suggests a possible molecular basis for non-prostate targeting of luciferase expression in the caput epididymis with sPSA promoter in sexually mature males as well as in bone in both pre-pubertal sexes. In mice, several androgen-dependent epididymal proteins such as cysteine-rich secretory protein-1 (Haendler et al. 1997, Schwidetzky et al. 1997), androgen-dependent glutathione peroxidase-like protein arMEP24 (Ghyselinck et al. 1993) and glutathione peroxidase 5 (Drevet et al. 1998) were mainly found in the epididymis of males, where the epididymal tissue-restricted and caput-regionalized Ets-like transcriptional factor PEA3 (polyoma enhancer activator 3) acted in co-operation with the $\mathrm{AR}$ controlling its tissue-specific expression. Likewise in humans a prostate epithelial-specific Ets transcriptional factor, PDEF, was recently reported to modulate PSA gene expression as a co-regulator of $\mathrm{AR}$ (Oettgen et al. 2000). Two putative Ets-binding sites are also found in the $440 \mathrm{bp}$ AREc region of sPSA promoter. These observations suggest that transgene expression in the caput epididymis of sPSA-Luc transgenic male mice might be mediated by PEA3 and/or other epididymalspecific Ets-like transcriptional factors in cooperation with AR to function on the AREc enhancer of sPSA promoter. The mechanism by which sPSA promoter is highly active in the growing bone but to a lesser extent in the adult bone is not understood. However, in early bone development the hematopoietic stem cells could employ certain Ets-specific transcription factors and/or AR that may be responsible for the induction of the sPSA promoter reporter activity found in bone tissues.

For the first time, our study was able to follow gene expression in target cells and tissues on a real-time basis using a non-invasive imaging modality. With respect to bone marrow luciferase reporter expression, we suggest the observed fluctuation and regional expression in live animals could reflect regional AR- or growth factorelicited activity in bone. Further work is needed to confirm or refute this suggestion.

Since luciferase imaging is quantitative, it provides an opportunity of assessing in a non-invasive manner the total number of tumor cells at the site of growth, the activity of the reporter gene(s) expressed by the target cells, and the ligand(s) responsible for activating the reporter activity in cancer cells, such as the concentrations of androgen and growth factors. With respect to the total number of cells, we do not know the lower detection limit for luciferase-tagged cells in transgenic animals, although others have reported that as few as 1000 transplanted cells bearing the luciferase gene could be quantitatively detected at an s.c. site (Edinger et al. 1999, Sweeney et al. 1999). However, a sensitivity study (Wu JC et al. 2001) that demonstrated bioluminescence imaging was predicted to be 100 - to 1000 -fold more sensitive than positron emission tomography in detection 
of reporter gene expression in mouse muscle and 10-fold in liver, indicating that luciferase imaging is well suited for quantitatively detecting tumor growth in vivo. With regard to concentrations of androgen and growth factors, the bioluminescent approach demonstrated in this communication could provide critical information on the concentration as well as the half-life of DHT in tissues. Assuming the intensity of luciferase imaging corresponds directly with the tissue level of DHT, using the non-invasive imaging approach we were able to calculate the half-life of tissue DHT in vivo to be $20 \mathrm{~h}$ by photon emission units, a result that has not been reported hitherto and which is consistent with the pharmacokinetic data of serum DHT (Wang et al. 1998, Baisley et al. 2002).

The sPSA-Luc transgenic animal model we have developed allows us to follow induction of the transgene over time using the IVIS imaging system. One advantage of this model is that it is now possible to study the kinetics of PSA induction in vivo in real time and correlate PSA kinetics in the mouse with tumor growth. This cannot be achieved using other reporter genes such as $\beta$-galactosidase and green fluorescent protein. Many factors including the number of luciferase-expressing cells, the promoter used to drive luciferase expression, the transgene copy number, the transgene integration site, and the distribution of luciferase-expressing cells can determine the sensitivity of bioluminescence imaging. A transgenic mouse line containing around 50 copies of MMP-13 promoter-driven luciferase transgene in its genome has been used to examine MMP-13 promoter activity throughout the process of wound healing in skin (Wu $\mathrm{N}$ et al. 2002). In our sPSA-Luc transgenic line, as few as two to five copies of luciferase transgene (Fig. 1b) allow us to visualize mouse prostate gland. This observation suggests that synergistic cooperation of the $\mathrm{pN} / \mathrm{H}$ fragment with the AREc enhancer may result in an extremely high transgene expression by sPSA promoter in target tissues, which agrees well with our previous in vitro study (Yeung et al. 2000) showing that sPSA promoter exhibits 2- to 3-fold higher activity than the WT PSA promoter in prostate cancer cell lines.

In summary, by combining the luciferase reporter system and the CGCD imaging technique we were able to quantitatively follow prostate development and the effects of androgen in activating sPSA promoter in living animals. The elucidation of the molecular mechanisms that regulate the tissue-specific expression of the PSA gene can provide important insights into prostate development and its neoplastic progression. Our data demonstrated that a small composite sPSA promoter is capable of driving a high level of expression of target gene reliably in the mouse prostate. The sPSA-Luc mouse model may be an ideal founder for the establishment of other bigenic imaging mouse models to study benign or malignant prostate growth. We are currently investigat- ing the feasibility of an sPSA-Luc model for assessing in vivo trafficking of prostate cancer metastasis by crossbreeding sPSA-Luc with the transgenic adenocarcinoma mouse prostate model (Hsieh et al. 2005).

\section{Acknowledgements}

We are grateful to Mr Gary Mawyer for editorial assistance. This work was supported financially in part by NIH Grant 1PO1CA098912-01 (to L W K C) and DOD Grant DAMD17-03-1-0160 (to G-L H). All authors declare that there is no conflict of interest that would prejudice its impartiality.

\section{References}

Adams JY, Johnson M, Sato M, Berger F, Gambhir SS, Carey M, Iruela-Arispe ML \& Wu L 2002 Visualization of advanced human prostate cancer lesions in living mice by a targeted gene transfer vector and optical imaging. Nature Medicine 8 891-897.

Allison J, Zhang YL \& Parker MG 1989 Tissue-specific and hormonal regulation of the gene for rat prostatic steroid-binding protein in transgenic mice. Molecular and Cellular Biology 9 2254-2257.

Baisley KJ, Boyce MJ, Bukofzer S, Pradhan R \& Warrington SJ 2002 Pharmacokinetics, safety and tolerability of three dosage regimens of buccal adhesive testosterone tablets in healthy men suppressed with leuprorelin. Fournal of Endocrinology 175 813-819.

Bronson FH \& Desjardins C 1977 Reproductive failure in aged CBF1 male mice: interrelationships between pituitary gonadotropic hormones, testicular function, and mating success. Endocrinology 101 939-945.

Chung LW \& Auble K 1988 Characterization of fetal urogenital sinus-induced prostatic hyperplasia in the mouse: time course, hormonal requirement, age dependency, and responsiveness of various adult organs to growth induction by fetal urogenital sinus tissues. Biology of Reproduction 39 50-57.

Clements JA 1989 The glandular kallikrein family of enzymes: tissue-specific expression and hormonal regulation. Endocrinology Revierws $10393-419$.

Cleutjens KB, van der Korput HA, van Eekelen CC, van Rooij HC, Faber PW \& Trapman J 1997a An androgen response element in a far upstream enhancer region is essential for high, androgen-regulated activity of the prostate-specific antigen promoter. Molecular Endocrinology 11 148-161.

Cleutjens KB, van der Korput HA, Ehren-van Eekelen CC, Sikes RA, Fasciana C, Chung LW \& Trapman J 1997b A 6-kb promoter fragment mimics in transgenic mice the prostate-specific and androgen-regulated expression of the endogenous prostate-specific antigen gene in humans. Molecular Endocrinology 11 $1256-1265$

Contag PR, Olomu IN, Stevenson DK \& Contag CH 1998 Bioluminescent indicators in living mammals. Nature Medicine 4 245-247.

Drevet JR, Lareyre JJ, Schwaab V, Vernet P \& Dufaure JP 1998 The PEA3 protein of the Ets oncogene family is a putative transcriptional modulator of the mouse epididymis-specific glutathione peroxidase gene gpx5. Molecular Reproduction and Development 49 131-140.

Edinger M, Sweeney TJ, Tucker AA, Olomu AB, Negrin RS \& Contag CH 1999 Noninvasive assessment of tumor cell proliferation in animal models. Neoplasia $1303-310$. 
Gabril MY, Onita T, Ji PG, Sakai H, Chan FL, Koropatnick J, Chin JL, Moussa M \& Xuan JW 2002 Prostate targeting: PSP94 gene promoter/enhancer region directed prostate tissue-specific expression in a transgenic mouse prostate cancer model. Gene Therapy 9 1589-1599.

Garabedian EM, Humphrey PA \& Gordon JI 1998 A transgenic mouse model of metastatic prostate cancer originating from neuroendocrine cells. PNAS 95 15382-15387.

Ghyselinck NB, Dufaure I, Lareyre JJ, Rigaudiere N, Mattei MG \& Dufaure JP 1993 Structural organization and regulation of the gene for the androgen-dependent glutathione peroxidase-like protein specific to the mouse epididymis. Molecular Endocrinology 7 $258-272$.

Greenberg NM, DeMayo FJ, Sheppard PC, Barrios R, Lebovitz R, Finegold M, Angelopoulou R, Dodd JG, Duckworth ML, Rosen JM et al. 1994 The rat probasin gene promoter directs hormonally and developmentally regulated expression of a heterologous gene specifically to the prostate in transgenic mice. Molecular Endocrinology 8 230-239.

Greenberg NM, DeMayo F, Finegold MJ, Medina D, Tilley WD, Aspinall JO, Cunha GR, Donjacour AA, Matusik RJ \& Rosen JM 1995 Prostate cancer in a transgenic mouse. PNAS 92 3439-3443.

Haendler B, Habenicht UF, Schwidetzky U, Schuttke I \& Schleuning WD 1997 Differential androgen regulation of the murine genes for cysteine-rich secretory proteins (CRISP). European Fournal of Biochemistry 250 440-446.

Hogan B, Beddington R, Costantini F \& Lacy E 1994 Manipulating the mouse embryo: A laboratory manual, edn 2. pp 217-252. Eds Cold Spring Harbor, NY: Cold Spring Harbor Laboratory Press.

Honigman A, Zeira E, Ohana P, Abramovitz R, Tavor E, Bar I, Zilberman Y, Rabinovsky R, Gazit D, Joseph A et al. 2001 Imaging transgene expression in live animals. Molecular Therapy 4 239-249.

Hsieh CL, Xie Z, Martin WD, Yu J, Amin M, Wu GJ \& Chung LW 2005 Real-time visualization of primary tumor growth and metastasis by bioluminescence imaging in a new transgenic mouse model of prostate cancer. Fournal of Urology 173561.

Kasper S, Sheppard PC, Yan Y, Pettigrew N, Borowsky AD, Prins GS, Dodd JG, Duckworth ML \& Matusik RJ 1998 Development, progression, and androgen-dependence of prostate tumors in probasin-large $\mathrm{T}$ antigen transgenic mice: a model for prostate cancer. Laboratory Investigation $78 \mathrm{i}-\mathrm{xv}$.

Leav I, Schelling KH, Adams JY, Merk FB \& Alroy J 2001 Role of canine basal cells in prostatic post natal development, induction of hyperplasia, sex hormone-stimulated growth; and the ductal origin of carcinoma. Prostate 47 149-163.

Marker PC, Dahiya R \& Cunha GR 2003 Spontaneous mutation in mice provides new insight into the genetic mechanisms that pattern the seminal vesicles and prostate gland. Developmental Dynamics 226 643-653.

Maroulakou IG, Anver M, Garrett L \& Green JE 1994 Prostate and mammary adenocarcinoma in transgenic mice carrying a rat C3(1) simian virus 40 large tumor antigen fusion gene. PNAS 91 $11236-11240$.

Oettgen P, Finger E, Sun Z, Akbarali Y, Thamrongsak U, Boltax J, Grall F, Dube A, Weiss A, Brown L et al. 2000 PDEF, a novel prostate epithelium-specific ets transcription factor, interacts with the androgen receptor and activates prostate-specific antigen gene expression. Fournal of Biological Chemistry 275 1216-1225.

Perez-Stable C, Altman NH, Brown J, Harbison M, Cray C \& Roos BA 1996 Prostate, adrenocortical, and brown adipose tumors in fetal globin/T antigen transgenic mice. Laboratory Investigation $\mathbf{7 4}$ 363-373

Price D 1963 Comparative aspects of development and structure in the prostate. In Biology of the Prostate and Related Tissues, pp 1-28. Eds EP Vollmer \& G Kauffmann. Washington, DC: US Government Printing Office.
Putz O, Schwartz CB, Kim S, LeBlanc GA, Cooper RL \& Prins GS 2001 Neonatal low- and high-dose exposure to estradiol benzoate in the male rat: I. Effects on the prostate gland. Biology of Reproduction 65 1496-1505.

Rehemtulla A, Stegman LD, Cardozo SJ, Gupta S, Hall DE, Contag CH \& Ross BD 2000 Rapid and quantitative assessment of cancer treatment response using in vivo bioluminescence imaging. Neoplasia 2 491-495.

Rehemtulla A, Hall DE, Stegman LD, Prasad U, Chen G, Bhojani MS, Chenevert TL \& Ross BD 2002 Molecular imaging of gene expression and efficacy following adenoviral-mediated brain tumor gene therapy. Molecular Imaging 1 43-55.

Sambrook J, Fritsch EF \& Mariatis T 1989 Molecular cloning: a laboratory manual, edn 2, pp 9.31-9.58. Eds Cold Spring Harbor NY: Cold Spring Harbor Laboratory Press.

Schaffner DL, Barrios R, Shaker MR, Rajagopalan S, Huang SL, Tindall DJ, Young CY, Overbeek PA, Lebovitz RM \& Lieberman MW 1995 Transgenic mice carrying a PSArasT24 hybrid gene develop salivary gland and gastrointestinal tract neoplasms. Laboratory Investigation 72 283-290.

Schuur ER, Henderson GA, Kmetec LA, Miller JD, Lamparski HG \& Henderson DR 1996 Prostate-specific antigen expression is regulated by an upstream enhancer. Fournal of Biological Chemistry $2717043-7051$.

Schwidetzky U, Schleuning WD \& Haendler B 1997 Isolation and characterization of the androgen-dependent mouse cysteine-rich secretory protein-1 (CRISP-1) gene. Biochemistry Fournal 321 325-332.

Shappell SB, Masumori N, Thomas T, Case T, Pau M, Kasper S \& Matusik RJ 2003 Transgenic mouse models of prostate carcinoma: Anatomic, histopathologic, and molecular considerations. In Prostate Cancer: Scientific and Clinical Aspects: Bridging the Gap, pp 245-319. Eds EN Lalani \& PD Abel. London: Imperial College Press.

Shappell SB, Thomas GV, Roberts RL, Herbert R, Ittmann MM, Rubin MA, Humphrey PA, Sundberg JP, Rozengurt N, Barrios R et al. 2004 Prostate pathology of genetically engineered mice: definitions and classification. The consensus report from the Bar Harbor meeting of the Mouse Models of Human Cancer Consortium Prostate Pathology Committee. Cancer Research 64 2270-2305.

Steers WD 20015 alpha-reductase activity in the prostate. Urology 58 17-24; discussion 24.

Sweeney TJ, Mailander V, Tucker AA, Olomu AB, Zhang W, Cao Y, Negrin RS \& Contag CH 1999 Visualizing the kinetics of tumor-cell clearance in living animals. PNAS 96 12044-12049.

Vaarala MH, Porvari KS, Kellokumpu S, Kyllonen AP \& Vihko PT 2001 Expression of transmembrane serine protease TMPRSS2 in mouse and human tissues. Fournal of Pathology 193 134-140.

Vooijs M, Jonkers J, Lyons S \& Berns A 2002 Noninvasive imaging of spontaneous retinoblastoma pathway-dependent tumors in mice. Cancer Research 62 1862-1867.

Wang C, Iranmanesh A, Berman N, McDonald V, Steiner B, Ziel F, Faulkner SM, Dudley RE, Veldhuis JD \& Swerdloff RS 1998 Comparative pharmacokinetics of three doses of percutaneous dihydrotestosterone gel in healthy elderly men - a clinical research center study. Foumal of Clinical Endocrinology and Metabolism $832749-2757$.

Wei C, Willis RA, Tilton BR, Looney RJ, Lord EM, Barth RK \& Frelinger JG 1997 Tissue-specific expression of the human prostate-specific antigen gene in transgenic mice: implications for tolerance and immunotherapy. PNAS 94 6369-6374.

Wu JC, Sundaresan G, Iyer M \& Gambhir SS 2001 Noninvasive optical imaging of firefly luciferase reporter gene expression in skeletal muscles of living mice. Molecular Therapy 4 297-306.

Wu JC, Inubushi M, Sundaresan G, Schelbert HR \& Gambhir SS 2002 Optical imaging of cardiac reporter gene expression in living rats. Circulation 105 1631-1634. 
Wu N, Opalenik S, Liu J, Jansen ED, Giro MG \& Davidson JM 2002 Real-time visualization of MMP-13 promoter activity in transgenic mice. Matrix Biology 21 149-161.

Xie X, Luo Z, Slawin KM \& Spencer DM 2004 The EZC-prostate model: noninvasive prostate imaging in living mice. Molecular Endocrinology 18 722-732.

Yeung F, Li X, Ellett J, Trapman J, Kao C \& Chung LW 2000 Regions of prostate-specific antigen (PSA) promoter confer androgen-independent expression of PSA in prostate cancer cells. Journal of Biological Chemistry 275 40846-40855.

Zhang J, Thomas TZ, Kasper S \& Matusik RJ 2000 A small composite probasin promoter confers high levels of prostatespecific gene expression through regulation by androgens and glucocorticoids in vitro and in vivo. Endocrinology 141 4698-4710.
Zhang L, Adams JY, Billick E, Ilagan R, Iyer M, Le K, Smallwood A, Gambhir SS, Carey M \& Wu L 2002 Molecular engineering of a two-step transcription amplification (TSTA) system for transgene delivery in prostate cancer. Molecular Therapy 5 223-232.

Zhang L, Johnson M, Le KH, Sato M, Ilagan R, Iyer M, Gambhir SS, Wu L \& Carey M 2003 Interrogating androgen receptor function in recurrent prostate cancer. Cancer Research 63 $4552-4560$.

Received 18 May 2005

Accepted 10 June 2005

Made available online as an Accepted Preprint 27 June 2005 